\title{
Commentary
}

\section{Issues in exploring variation in childhood socioeconomic gradients by age: A response to Case, Paxson, and Vogl}

\author{
Edith Chen $^{\mathrm{a}, *}$, Andrew D. Martin ${ }^{\mathrm{b}}$, Karen A. Matthews ${ }^{\mathrm{c}}$ \\ ${ }^{a}$ Department of Psychology, University of British Columbia, 2136 West Mall, Vacouver, BC, Canada V6T 1Z4 \\ ${ }^{\mathrm{b}}$ Washington University, USA \\ ${ }^{\mathrm{c}}$ School of Medicine, University of Pittsburgh, USA
}

Available online 28 November 2006

The issue of how best to study relationships of socioeconomic status (SES) with health during childhood is an important one, and Case, Paxson, and Vogl (this issue) very articulately describe how different decisions related to sample composition and variable calculations can affect statistical results. These decisions are not merely academic; rather, they have implications for policy and practice, given that evidence for stronger SES gradients in certain age groups would argue for interventions targeted at specific timepoints during childhood.

The question of when SES gradients are steepest in childhood has been an unresolved one (Chen, Matthews, \& Boyce, 2002). For example, some researchers have argued that gradients are stronger early in childhood, and weaken during adolescence as school influences create an equalizing effect on health (West \& Sweeting, 2004). Others have argued that effects of SES accumulate over time to predict health in young adulthood (Power, Manor, \& Matthews, 1999), and thus that childhood gradients become stronger with age (Case, Lubotsky, \& Paxson, 2002). Our study found that associations of lower SES with poorer child health did not vary by age for overall health ratings, activity limitations,

*Corresponding author.

E-mail address: echen@psych.ubc.ca (E. Chen). and school limitations, but did vary by age for injury and acute respiratory illness, such that lower SES was associated with higher morbidity rates during adolescence only (Chen, Martin, \& Matthews, 2006).

Our study investigated five health outcomes, and concluded that relationships of SES with child health vary by age for acute conditions, but not for global health measures. Case et al. (this issue) primarily focus on one of these five outcomes in their commentary (overall health rating), and disagree with our conclusions. Specifically, they document that if one removes children who report themselves to be the household reference person (a group of 232 primarily 17- and 18-year olds), and one either expands the sample size by including additional years of data from the US National Health Interview Survey (NHIS), or changes the categorical income variable in the data set to one in which income values are assigned based on averages from the Current Population Surveys, then a significant age $\times$ income interaction effect emerges for overall rating of child health.

With respect to the issue of inclusion criteria, the authors raise an important methodological issue. We chose to retain all children ages $0-18$ in our sample because this allowed our sample to be most generalizable to the population and because we did not want to make inferences about the 
characteristics of a subgroup that we were not able to confirm (e.g., that many 18-year olds who report themselves to be the household reference person would be enrolled in college). While we agree with Case et al. (this issue) that if 18-year olds are enrolled in college, their "current household income will be a poor guide to the SES of the households in which they were raised," it is also important to note that because this data set is cross-sectional, we were drawing conclusions only about concurrent associations of SES with health, and not about lifetime history of SES and its relationship to health. In addition, we find that Case, Paxson, and Vogl's recommendation of including only dependents (children who do not report themselves to be the household reference person) does not produce clearcut findings. When we repeat our analyses including only dependents, we find that all of the main effects for global health measures remain.

That is, lower family income remained significantly associated with a greater likelihood of fair/ poor health $(B=-.070, \mathrm{SE}=.004, p<.001)$, activity limitations $(B=-.046, \mathrm{SE}=.003, p<.001)$, and school limitations $(B=-.048, \mathrm{SE}=.004, p<.001)$, and no significant interactions effects of age $\times$ income were found (fair/poor health: $B=-.0007$, $\mathrm{SE}=.0007$, n.s.; activity limitations: $B=.0003$, $\mathrm{SE}=.0005$, n.s.; school limitations: $B=.0000$, $\mathrm{SE}=.0009$, n.s.). In addition, Case, Paxson, and Vogl report that the majority of children who reported themselves to be the household reference person were 18 years old. When we repeated our analyses including only $0-17$-year olds, we found the same patterns of a main effect of income predicting fair/poor health $(B=-.071, \mathrm{SE}=.004$, $p<.001)$, activity limitations $(B=-.047, \mathrm{SE}=$ $.003, p<.001)$, and school limitations $(B=-.047$, $\mathrm{SE}=.004, p<.001)$, but no significant interactions with age (fair/poor health: $B=-.0011, \mathrm{SE}=.0008$, n.s.; activity limitations: $B=.0001, \mathrm{SE}=.0005$, n.s.; school limitations: $B=.0002, \mathrm{SE}=.0009$, n.s.). It is only when one combines the exclusion of those who reported themselves to be the household reference person (independents) with either additional years of data from the NHIS or with changing how the income variable is coded that one gets a significant interaction effect. Or, if one changes the dependent variable from our definition (odds of fair/poor health) to a 5-point scale, and then combines excluding independents with this new dependent variable using ordered probits, one gets a significant interaction effect. To us, this suggests that the effect of removing independents is not robust.

Case, Paxson, and Vogl also report an alternative approach to coding income. The NHIS data set contains income as a categorical variable. Case, Paxson, and Vogl used data from the March Current Population Surveys to impute the mean household income for each income category. Neither approach is ideal, as the constraints of the data set were such that family income as a dollar amount was not ascertained. In light of this limitation, both approaches - using income as a categorical variable and imputing income values based on a separate data set - seem reasonable, and in fact produce similar patterns of results overall.

Finally, it is important to note that the earlier Case et al. (2002) paper utilized a different sample, as they reported NHIS data across the years 1986-1995, whereas our paper reported on the 1994 NHIS sample (which contained the largest single year cohort of children). Thus, the two sets of analyses are not based on the same data source (in that there are different people in each, with only approximately $1 / 10$ of the sample overlapping), and it is perhaps not surprising that findings are not identical.

In sum, Case, Paxson, and Vogl provide some thoughtful examples of how decisions to approach data can affect statistical results. Some of these issues are difficult ones, with no clear-cut solutions, given the limitations of large survey data, which often do not contain information with the level of specificity one would ideally like. Our findings provide a first step toward understanding age differences in concurrent associations of SES with child health. We wholeheartedly agree with their conclusion that longitudinal studies will provide much more definitive answers to the question of how SES relationships change as children age.

\section{References}

Case, A., Lubotsky, D., \& Paxson, C. (2002). Economic status and health in childhood: The origins of the gradient. American Economic Review, 92, 1308-1334.

Case, Paxson, \& Vogl, this issue. Socioeconomic status and health in childhood: A comment on Chen, Martin and Matthews, "Socioeconomic status and health: Do gradients differ within childhood and adolescence?", in press, doi:10.1016/j.socscimed.2006.10.025.

Chen, E., Matthews, K. A., \& Boyce, W. T. (2002). Socioeconomic differences in children's health: How and why do 
these relationships change with age? Psychological Bulletin, 128, 295-329.

Chen, E., Martin, A. D., \& Matthews, K. A. (2006). Socioeconomic status and health: Do gradients differ within childhood and adolescence? Social Science \& Medicine, 62, 2161-2170.
Power, C., Manor, O., \& Matthews, S. (1999). The duration and timing of exposure: Effects of socioeconomic environment on adult health. American Journal of Public Health, 89, 1059-1065.

West, P., \& Sweeting, H. (2004). Evidence on equalisation in health in youth from the West of Scotland. Social Science \& Medicine, 59, 13-27. 